Rev. Elev. Méd. vét. Pays trop., 1972, 25 (2) : 205-218

\title{
Enquête sur la pathologie de la faune sauvage en Afrique centrale \\ Le parasitisme des Suidés sauvages Premiers résultats d'enquête
}

par P. M. TRONCY (*), M. GRABER (**) et J. THAL (*)

\begin{abstract}
RESUME
Les auteurs dénombrent les espèces parasites des Suidés sauvages, recueillies en Afrique centrale, au cours d'une enquête sur la pathologie de la faune cynégétique. Quatre espèces nouvelles ont été déterminćes et décrites dans une autre publication.

Le potamochère est un hôte nouveau pour une espèce de Nématode, le phacochère pour une espèce de Nématode et deux espèces de Trématodes et l'hylochère pour cinq espèces de Nématodes, une espèce de Trématode et une espèce de Cestode.
\end{abstract}

En Afrique centrale existe une zone cynégétique de grande valeur, où la densité humaine - et partant, la densité des animaux domestiques - est extrêmement faible (carte $n^{\circ} \mathrm{I}$ ).

La variété des climats (soudanien du septième au onzième parallèle, guinéen plus au sud), l'importance du réseau hydrographique, y favorisent la multiplication des animaux sauvages : de ce fait, ils représentent pour ce pays un potentiel économique important qu'il importe de sauvegarder.

Les premiers résultats d'une enquête concernant la pathologie des animaux de chasse de cette région permettent, d'ores et déjà, de préciser la nature et l'importance du parasitisme des Suidés sauvages: phacochères ( $P h a-$ cochoerus aethiopicus, Pallas 1766), potamochères (Potamochoerus porcus, Cuvier 1826)

(*) I.E.M.V.T., Laboratoire de Farcha, B.P. n* 433, Fort-Lamy, Tchad.

(**) Laboratoire de Parasitologie, Ecole vétérinaire, 23, quai Chauveau, Lyon, France. et hylochères (Hylochoerus meinerizageni, Thomas 1904).

Les phacochères sont abondants dans toute la zone cynégétique : ce sont des animaux de sayane que l'on rencontre partout au nord du neuvième parallèle. Plus au sud, on les trouve hors des galeries forestières.

Les potamochères n'apparaissent qu'en deçà du huitième parallèle et ils deviennent de plus en plus nombreux au fur et à mesure que l'on s'avance vers l'équateur : ce sont, en général, des animaux forestiers, bien que, dans certains cas, on puisse les observer à découvert.

Les hylochères, dont la présence a été suspectée au-delà du septième parallèle, ne sont vraiment connus qu'au sud du sixième parallèle, c'est-à-dire dans la grande forêt équatoriale.

Au-dessous du septième parallèle, les contacts entre phacochères, potamochères et hylochères sont donc possibles, comme le montre 


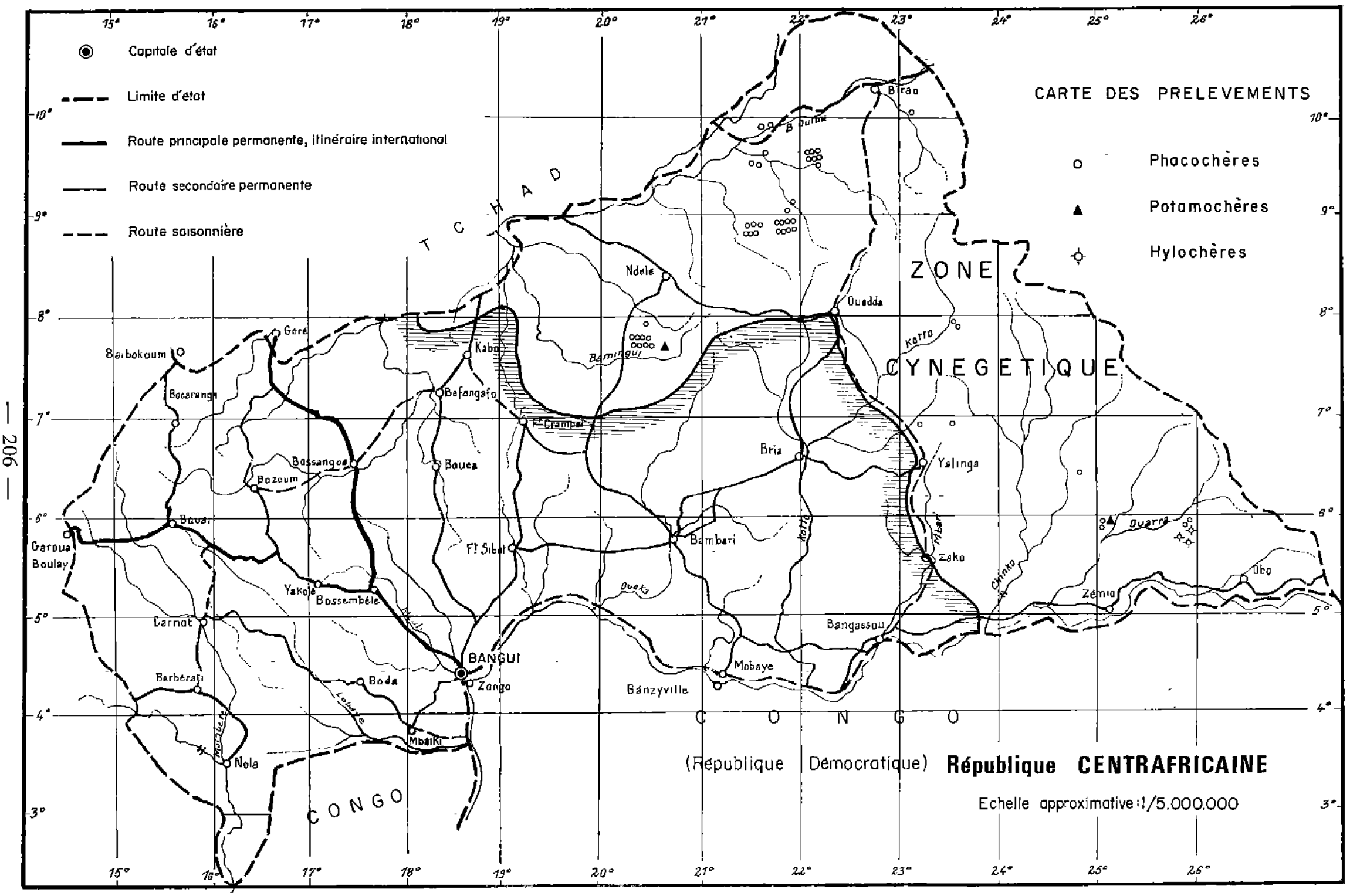


la carte $\mathrm{n}^{\circ} \mathrm{I}$ où sont portés les lieux de récoltes et d'autopsies.

La coexistence, dans un même biotope, de plusieurs espèces de Suidés proches les uns des autres, pose le problème des interférences entre leurs faunes parasitaires respectives. Sans prétendre fournir une vue exhaustive sur cette question, le présent travail a, en particulier, pour but de mettre en évidence les relations étroites qu'entraîne la promiscuité d'animaux proches du point de vue systématique et possédant des exigences écologiques sensiblement différentes.

\section{HELMINTHES RENCONTRES}

\section{NEMATODES (*)}

\section{ORDRE DES ENOPLIDA}

\subsection{Superfamille des Trichuroides}

1.1.1. Famille des Trichinellidae Ward, 1907 Genre Trichinella Railliet, 1895.

Trichinella spiralis (Owen, 1835) Railliet, 1895.

La forme larvaire de Trichinella spiralis a été rencontrée à plusieurs reprises chez le phacochère au Sénégal (26) et chez le potamochère au Kenya (36).

En République Centrafricaine, le Nématode a été recherché systématiquement chez 30 phacochères, un potamochère et un hylochère. Les examens sont tous demeurés négatifs. Le même phénomène a été observé au Tchad (23).

\section{ORDRE DES STRONGYLIDA}

\subsection{Superfamille des Strongyloidea}

2.1.1. Famille des Synganidae Leiper, 1912 Sous-famille des Stephanurinae Railliet, Henry et Bauche, 1919.

Genre Stephanurus Diesing, 1839.

Stephanurus dentatus Diesing, 1839.

Hôte: Phacochoerus aethiopicus (deux cas sur 70 examens). Le phacochère est un hôte nouveau (26).

(*) La classification retenue est celle de Roman et Chabaud, in «Traité de Zoologie » de Grassé: Nemathelminthes, 1965, IV, Fasc. 2, 705-13 et Fasc. 3, 869-1.200.
Localisations :

- Foie (une femelle);

- Tissu adipeux périrénal (un mâle).

Répartition (Coordonnées I.B.A.H.) : 23.6. C.c. et 21.8.D.d. Les deux animaux infestés ont été abattus à $300 \mathrm{~km}$ l'un de l'autre.

Rôle pathogène : la migration des larves de Stephanurus dans l'organisme des Suidés provoque diverses réactions inflammatoires qui passent, en général, inaperçues. Cependant, en cas de parasitisme massif, le Nématode est la cause d'un amaigrissement sensible avec cachexie, pouvant entraîner la mort de l'animal. Parfois des signes nerveux apparaissent: crises rabiformes, parésies ou paralysies, en rapport avec les migrations larvaires.

2.1.2. Famille des Strongylidae Baird, 1853

2.1.2.1. Sous-famille des Oesophagostominae Railliet, 1915

2.1.2.1.1. Genre Bourgelatia Railliet, Henry et Bauche, 1910

- Bourgelatia pricei (Schwartz, 1928) N. Comb.

Hôtes: Phacochoerus aethiopicus (10 cas sur 48); Hylochoerus meinertzageni (un cas sur trois).

Localisation : gros intestin.

Répartition: Phacochère, 20.7.C.b., 21.8. D.c., 21.8.D.d., 24.6.B.d., 25.5.C.c. et 25.5. D.d.; Hylochère, 25.5.D.d.

La grande ressemblance de ce ver (bourse caudale du mâle, extrémité caudale de la femelle) avec le genre Phacochoerostrongylus Schwartz, 19.28 a conduit à. une étude comparée, d'où il ressort que le genre Phacochoerostrongylus est synonyme du genre Bourgelatia (52).

Si le parasitisme par Bourgelatia pricei est très fréquent, les infestations sont, par contre, légères.

- Bourgelatia hylochoeri Van den Berghe, 1943.

Hôte: Hylochoerus meinertzageni (un cas sur trois).

Tocalisation: gros intestin.

Répartition : 25.5.D.d. 
Trois femelles ont été examinées (52), dont une en mauvais état.

Rôle pathogène des Bourgelatia: inconnu. Par analogie avec les Esophagostomes et les Trichonèmes qui appartiennent à des genres voisins, les Bourgelatia pourraient être pathogènes au stade larvaire.

\subsection{Genre Daubneya Le Roux, 1940}

Dix espèces ont été dénombrées, dont trois nouvelles. Leur description a fait l'objet d'une étude spéciale (52), accompagnée d'une importante iconographie et d'une clef de détermination des espèces.

Ce sont :

\section{- Daubneya centrafricanum n.Sp.} 48).

Hôte: Phacochoerus aethiopicus (13 cas sur

Localisation: gros intestin.

Répartition : 20.7.C.b., 21.8.D.c., 21.8.D.d., 21.9.B.b, 21.9.D.c., 22.9.C.c.

Ce nouvel Fsophagostome se différencie des autres espèces par ses dimensions générales, l'aspect de la capsule buccale et l'extrémité postérieure de la femelle. C'est un parasite assez répandu.

- Daubneya euzebyi n.Sp.

Hôte : Hylochoerus meinertzageni (deux cas sur trois).

Localisation : gros intestin.

Répartition : 25.5.D.d.

Représenté seulement par trois femelles, il se caractérise par son extrémité céphalique, la forme de l'œsophage et l'extrémité caudale de la femelle.

- Daubneya eurycephalum Goodey, 1924.

Hôte: Phacochoerus aethiopicus (14 cas sur 48).

Localisation: gros intestin.

Répartition: 20.7.C.b., 21.8.D.c. et 21.8 . D.d., 21.9.B.b et 21.9.D.a., 22.9.C.a.

L'extrémité antérieure est typique, ce qui rend aisée 1'identification de cet CEphagostome.

- Daubneya farchai n.Sp.

Hôtes: Phacochoerus aethiopicus (un cas sur 48); Hylochoerus meinertzageni (deux cas sur trois).

Localisation: gros intestin.

Répartition : 25.5.D.d.

Cet Esophagostome nouveau dont la capsule buccale est caractéristique semble plus spécialement lié à l'hylochère qui vit habituellement en zone forestière où a été abattu le seul phacochère parasité.

Par ailleurs, le nombre d'exemplaires recueillis est faible : deux femelles chez le phacochère; un mâle et une femelle chez le premier hylochère; deux mâles et trois femelles chez le second.

- Daubneya goodeyi Daubney, 1926.

Hôtes: Phacochoerus aethiopicus (31 cas sur 48); Hylochoerus meinertzageni (un cas sur trois).

Localisation: gros intestin.

Répartition : toute la zone cynégétique explorée.

L'extrémité postérieure de la femelle (vulve sur une excroissance terminale) et la longueur des spicules du mâle facilitent l'identification du parasite.

Chez le phacochère, c'est un parasite fréquent et abondant.

L'hylochère est un hôte nouveau: le seul animal porteur hébergeait huit mâles et huit femelles.

- Daubneya mwanzee Daubney, 1924.

Hôtes: Phacochoerus aethiopicus (33 cas sur 48); Hylochoerus meinertzageni (un cas sur trois).

Localisation : gros intestin.

Répartition : toute la zone cynégétique explorée.

Daubneya mwanzee est un parasite du phacochère. L'hylochère est un hôte nouveau.

- Daubneya mpwapwae Duthy, 1947.

Hôte: Phacochoerus aethiopicus (5 cas sur 48).

Localisation: gros intestin. 

D.d.

Répartition: 20.7.C.b., 21.8.D.c. et 21.8.

La longueur des spicules chez le mâle, la longueur du vagin chez la femelle permettent la diagnose de cette espèce.

Le parasite est rare et les infestations légères.

- Daubneya roubaudi Daubney, 1926.

Hôte: Phacochoerus aethiopicus (25 cas sur 48).

Localisation : gros intestin.

Répartition: toute la zone cynégétique.

Ce ver, très voisin de Daubneya mwanzee, ne présente toutefois pas de valvule csophagienne. On le distingue facilement par la forme particulière du mucron des papilles céphaliques qui offre une sorte de plicature. Un complément iconographique en précise l'anatomie (52).

- Daubneya simpsoni Goodey, 1924.

Hôte: Phacochoerus aethiopicus (5 cas sur 48).

Localisation: gros intestin.

Répartition: toute la zone cynégétique.

L'aspect court et massif de l'œsophage et l'extrémité postérieure de la femelle constituent de bons éléments de différenciation.

C'est un parasite assez rare. Le niveau de l'infestation est faible.

- Daubneya yorkei Thornton, 1924.

Hôtes: Phacochoerus aethiopicus (27 cas sur 48); Hylochoerus meinertzageni (deux cas sur trois).

Localisation: gros intestin.

Répartition: toute la zone cynégétique.

Daubneya yorkei est assez difficile à reconnaître au sein d'une population comprenant plusieurs espèces d'œsophagostomes associés, ce qui est souvent le cas. Son identification est possible en tenant compte des caractères suivants : papilles céphaliques très petites, faibles dimensions des spicules chez le mâle et du vagin chez la femelle.

Chèz le phacochère, il s'agit d'un Esophagostominae relativement fréquent et assez abondant.
L'hylochère, qui est un hôte nouveau, semble peu parasité : ont été comptés trois mâles et six femelles dans le premier cas et une femelle seulement dans le second.

- Rôle pathogène des Esophagostomes.

Les phacochères (Dr Thal, communication personnelle) porteurs d'un grand nombre d'Esophagostomes sont dans un état de grande maigreur, voire d'extrême misère physiologique (c'est le cas d'un jeune de six mois). Cependant, les différentes autopsies effectuées n'ont pas permis de constater la présence de nodules d'Esophagostomose larvaire. Peut-être s'agit-il d'une observation insuffisante, car, par analogie avec ce que l'on voit chez les porcs domestiques atteints d'CEsophagostomose à Oesophagostomum dentatum ou Esophagostomum quadrispinulatum, on peut penser que ce sont les larves qui déterminent les symptômes décrits ci-dessus.

\subsection{Genre Murshidia Lane, 1914}

Sous-genre Chabaudia Campana-Rouget, 1959

- Murshidia (Chabaudia) hamata Daubney, 1923.

Hôtes: Phacochoerus aethiopicus (un cas sur 48); Hylochoerus meinertzageni (un cas sur trois).

Localisation : gros intestin.

Répartition : 25.5.D.d.

La diagnose de l'espèce repose sur deux caractères, faciles à mettre en évidence: le bourrelet et périvulvaire de la femelle et les spicules spatulés du mâle.

Il semblerait, en considérant les pourcentages d'infestation, que Murshidia (Chabaudia) hamata soit surtout un parasite d'hylochère.

- Murshidia (Chabaudia) pugnicaudata (Leiper, 1909) Yorke et Maplestone, 1926.

Hôtes: Phacochoerus aethiopicus (un cas sur 48); Hylochoerus meinertzageni (deux cas sur trois).

Localisation: gros intestin.

Répartition : 25.5.D.d.

Cette espèce se caractérise par l'extrémité postérieure de la femelle et les spicules du 
mâle. Comme dans le cas de Murshidia (Chabaudia) hamata, l'unique phacochère parasité a été tué en zone de forêt: il ne présentait qu'un seul ver (un mâle).

L'hylochère est un hôte nouveau et il est paradoxal de constater que deux hylochères sur trois hébergeaient Murshidia (Chabaudia) pugnicaudata, alors que le Nématode est classiquement considéré comme un parasite de phacochère.

- Rôle pathogène des Murshidia.

Inconnu. Probablement identique à celui des Esophagostomes.

\subsection{Superfamille des Ancylostomatoidea}

2.2.1. Famille des Ancylostomatidae Looss, 1905

Sous-famille des Globocephalinae Travassos et Vogelsang, 1922

Genre Globocephalus Molin, 1861

Globocephalus urosubulatus Alessandrini, 1909

Hôte: Potamochoerus porcus (un cas sur deux).

Localisation: gros intestin.

Répartition : 25.5.C.c.

La systématique du genre Globocephalus est imprécise. Elle se fonde sur la longueur des spicules chez le mâle (39). Ceux-ci, dans le matériel examiné, sont susceptibles de présenter d'importantes variations de taille, allant de $0,290 \mathrm{~mm}$ à $0,590 \mathrm{~mm}$, en tous cas, jamais au-dessus de $0,600 \mathrm{~mm}$.

Les trois espèces de Globocephalus connues à ce jour en Afrique et à Madagascar chez le potamochère sont Globocephalus longemucronatus Molin, 1861 (Longueur des spicules : $0,690$ à $0,720 \mathrm{~mm})$, Globocephalus versteri Ortlepp, 1964 (Longueur des spicules : 0,830 à $0,850 \mathrm{~mm}$ ) et Globocephalus madagascariensis Chabaud, Bain et Houin, 1966.

Le potamochère est un hôte nouveau.

Rôle pathogène: ces vers sont hématophages. Chez le porc domestique, la globocéphalose se traduit par des signes entéritiques, de l'anémie, avec comme conséquence une altération de l'état général, d'autant plus accusée que les parasites sont plus nombreux.

\section{ORDRE DES ASCARIDIDA}

\subsection{Superfamille des Cosmocercoidea}

3.1.1. Famille des Atractidae (Railliet, 1917) Travassos, 1919

Genre Probstmayria Ransom, 1907

Probstmayria suis n.Sp.

Hôtes: Phacochoerus aethiopicus; Hylochoerus meinertzageni (un cas sur trois).

Localisation : gros intestin.

Répartition: toute la zone cynégétique.

Ce parasite nouveau a été décrit ailleurs (53). On le rencontre chez la plupart des phacochères autopsiés, bien qu'il n'ait jamais fait l'objet d'une recherche systématique (on le trouve, en effet, dans le mucus intestinal, mélangé à d'autres helminthes). Le Roux (31) signale la présence de Probstmayria vivipara chez des phacochères du Ghana et du Natal. Il est possible que, dans ce cas, il s'agisse de Probstmayria suis, Atractidae qui ne peut être distingué que par les mâles (existence d'un gubernaculum, spicules très inégaux, position différente des papilles caudales) et ceux-ci sont, dans l'intestin des Suidés, particulièrement rares.

Rôle pathogène: on admet que, chez les Equidés, le rôle pathogène de Probstmayria vivipara est minime. Il en est sans doute de même pour Probstmayria suis.

Il est bon de noter que, les Probstmayria étant vivipares, un seul hôte est capable d'héberger des millions d'helminthes.

\subsection{Superfamille des Ascaridoidea}

3.2.1. Famille des Ascarididae Baird, 1853

Genre Ascaris Linné, 1758

Ascaris Phacochoeri Gedoelst, 1916

Hôte: Phacochoerus aethiopicus (17 cas sur 48).

Localisation: intestin grêle.

Répartition: toute la zone explorée.

D'après Sprent (50), Warren (57) et Kreis (29), Ascaris phacochoeri ne se différencie d'Ascaris suum que par les dimensions des spicules: ils mesurent en moyenne 600 a (exemplaires de R.C.A.), tandis que ceux d'Ascaris sum ont une longueur toujours 
supérieure à $1 \mathrm{~mm}$. Pour cette raison, nous estimons qu'il s'agit bien de l'espèce propre au phacochère.

Plus du tiers des animaux sont atteints, mais le niveau de l'infestation est faible : c'est ainsi que, sur huit jeunes abattus, un seul présentait un grand nombre d'Ascaris. Un autre sujet, très âgé celui-là, était aussi fortement infesté.

Rôle pathogène : semblable à celui d'Ascaris suum du porc.

\section{ORDRE DES SPIRURIDA}

\subsection{Superfamille des Spiruroidea}

4.1.1. Famille des Spiruridae Oerley, 1885

Genre Physocephalus Diesing, 1861

Physocephalus sexalatus (Molin, 1860) Diesing, 1861

Hôte : Phacochoerus aethiopicus (trois cas sur 48).

Localisation : estomac.

Répartition : 21.8.D.d., 22.9.C.a.

L'identification du parasite est facilitée par la structure de l'œsophage, le diagramme céphalique caractéristique et les ailes longitudinales dans le tiers antérieur du corps.

Deux phacochères examinés n'hébergeaient qu'un seul parasite; par contre, dans le troisième cas, l'infestation était massive.

Les deux zones où ont été récoltés les Physocephalus étaient suffisamment distantes l'une de l'autre pour que l'on puisse considérer que cette espèce est largement répandue en R.C.A.

Rôle pathogène : chez le porc domestique, lc ver provoque une gastrite parasitaire et, selon l'intensité du parasitisme, une perte d'appétit plus ou moins nette.

\subsection{Superfamille des Physalopteroidea}

4.2.1. Famille des Gnathostomatidae Railliet, 1895

Genre Gnathostoma Owen, 1836

Gnathostoma hispidum Fedschenko, 1871

Hôte: Potamochoerus porcus (un cas sur deux).

Localisation : estomac.
Répartition : 25.5.C.c.

Gnathostoma hispidum est un Nématode recouvert d'épines que l'on reconnaît facilement grâce à ses caractères généraux.

L'infestation était importante et les vers profondément implantés dans la muqueuse gastrique.

Le parasite n'a encore jamais été signalé chez le potamochère au nord de l'équateur, mais il existe chez le porc domestique (abattoir de Bangui).

Rôle pathogène : par sa structure cuticulaire épineuse et son insertion dans la muqueuse gastrique, c'est un Spiruridé très irritant et très pathogène, susceptible de déterminer une gastrite parasitaire grave avec formations de volumineux nodules dans l'estomac, et, parfois, des péritonites par perforation de l'organe.

4.2.2. Famille des Physalopteridae Leiper, 1908

Genre Physaloptera Rudolphi, 1819

Physaloptera joyeuxi Gendre, 1928

Hôte: Phacochoerus aethiopicus (14 cas sur 48).

Localisation : estomac.

Répartition : 21.8.D.c., 21.8.D d., 21.9.B.b., 22.9.C.a.

Cette espèce - Hexadelphe - correspond à la description de GENDRE (27) qui avait découvert ce Physaloptère au Dahomey.

En R.C.A., on le trouve chez les animaux abattus en zone de savane, au niveau et au nord du neuvième parallèle.

Le parasitisme est, en général, important.

Rôle pathogène: inconnu. Toutefois, les fortes infestations d'animaux en bon état d'entretien laissent supposer qu'il s'agit là d'un Nématode peu pathogène.

\subsection{Superfamille des Filaroidea}

4.3.1. Famille des Setariidae Yorke et Maplestone, 1926

Genre Setaria Viborg, 1795

- Setaria castroi Ortlepp, 1964

Hôte: Phacochoerus aethiopicus (deux cas sur 48). 
Localisation : cavité péritonéale.

Répartition : 21.9.D.a., 22.9.C.a.

L'un des phacochères était porteur de quatre Filaires et l'autre d'une seule. Cette espèce, décrite sous le nom de Setaria shohoi par DURETTE-DESSET (16), se caractérise par la structure de l'extrémité céphalique et des extrémités caudales des mâles et des femelles.

- Setaria congolensis Railliet et Henry, 1911 (*).

Hôtes: Potamochoerus porcus (deux cas sur deux); Hylochoerus meinertzageni (un cas sur trois).

Localisation : cavité péritonéale.

Répartition: 20.7.D.a., 25.5.C.c. et 25.5. D.d.

L'hylochère hébergeait cinq parasites et les deux potamochères, 5 et 3 respectivement.

Setaria congolensis, connue chez Sus scrofa domesticus, Sus cristatus et Potamochoerus porcus, n'avait encore jamais été signalée chez Hylochoerus meinertzageni qui représente un hôte nouveau. Ce fait confirme la relative ubiquité des Sétaires de Suidés qu'ORTLEPP (39) avait indiquée, en décrivant Setaria castroi simultanément chez le phacochère et chez le potamochère.

Rôle pathogène des Sétaires: minime dans toutes les espèces animales.

\section{CESTODES (**)}

\section{ORDRE DES CYCLOPHYLLIDEA}

1. Famille des Anoplocephalidae Cholodkowski, 1902, emended Fuhrmann, 1907

1.1. Sous-famille des Anoplocephalinae Blanchard, 1891, emended Fuhrmann, 1907

Genre Moniezia Blanchard, 1891

Moniezia mettami Baylis, 1934

Hôtes: Phacochoerus aethiopicus (17 cas sur 48); Hylochoerus meinertzageni (un cas sur trois).

(") Les spécimens ont été đéterminés par Madame Durette-Desset que nous tenons à remercier vivement.

(**) La classification est celle de Joyeux (Ch.) et Baer (J.G.) in Grassé «Traité de zoologie », 1961. t. IV, fasc. I, 347-560.
Localisation : intestin grêle (ileon).

Répartition: Phacochères, toute la zone cynégétique; Hylochère, 25.5.D.d.

Les exemplaires recueillis ont les caractères de l'espèce, tels qu'ils ont été décrits par BAYLIS (8) : dimensions générales, aspect des organes génitaux, longueur de la poche du cirre.

L'hylochère est un hôte nouveau: le seul animal atteint hébergeait deux parasites immatures.

Moniezia mettami est un Anoplocephalinae assez répandu dans l'Est de la R.C.A. La plupart du temps, le niveau de l'infestation est faible.

Rôle pathogène: inconnu.

2. Famille des Taeniidae Ludwig, $\mathbf{1 8 8 6}$

2.1. Genre Echinococcus Rudolphi, 1801

Echinococcus Sp.Forme larvaire (kyste hydatique)

Hôtes: Phacochoerus aethiopicus (28 cas sur 70) (***); Potamochoerus porcus (un cas sur deux).

Localisations : foie, rate, poumon et cœur.

Répartition : 19.8.D.b. et B.a.; 20.7.D.a.; 21.8.D.a., D.b. et D.d.; 21.8.C.d; 21.9.B.b. et B.d.; 21.9.D.c. et D.d.; 22.9.A.a.; 22.9.C.a.; 23.7.A.b.; 23.7.D.c., 23.6.C.c.; 24.6.B.b.; 25.5 . C.c.

Les cas les plus nombreux ont été observés aux points suivants: 21.9.B.b.; 22.9.C.a. et 23.6.C.c.

A cette liste, il y a lieu d'ajouter deux phacochères porteurs de kystes sur le foie et sur la rate et abattus sur le haut Vassako (20.7.C.b. et 20.7.C.d.).

Six autres présentaient des kystes non identifiables : il est possible, dans ces conditions, que des cas d'echinococcose soient passés inaperçus.

Une carte annexe permet de mieux situer les lieux de récolte.

Il apparaît donc que plus de 40 p. 100 des Suidés sauvages de R.C.A. présentent, à l'autopsie, des kystes hydatiques à divers stades

${ }^{(* * * *)}$ Résultats définitifs. 


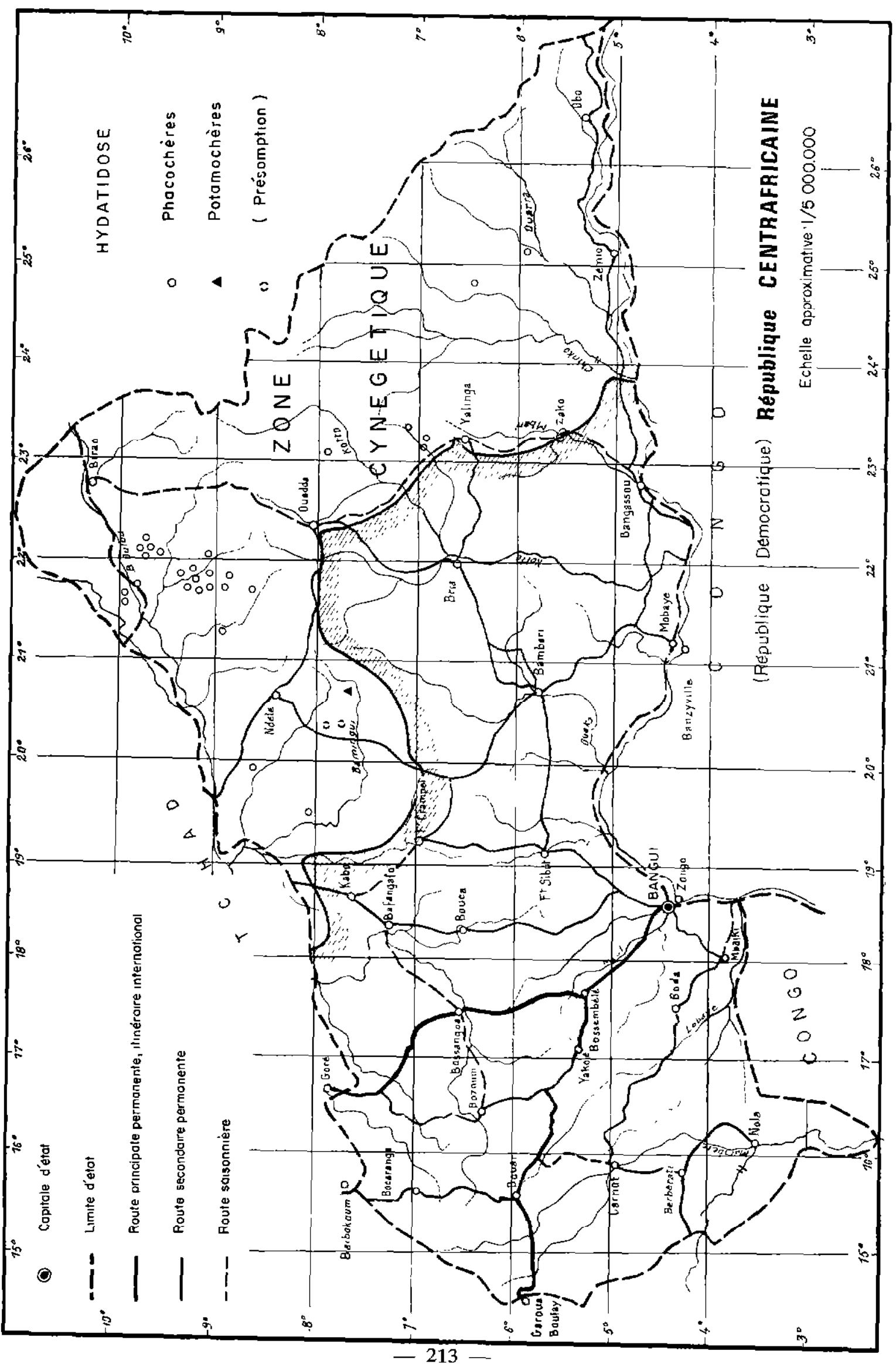


d'évolution. Leurs dimensions vont de 0,5 à $5 \mathrm{~cm}$ de diamètre. Ils sont souvent fertiles.

Par ailleurs, la présence simultanée sur la rivière Kapa (21.8.D.d.) et dans ses environs d'un lion, Panthera leo, Linné, porteur de Ténias echinocoques et de phacochères atteints d'hydatidose permet d'envisager l'existence, dans cette région de l'Afrique centrale, d'un cycle sylvatique ayant le phacochère ou le potamochère comme hôte intermédiaire principal.

Les cynhyènes, Lycaon pictus, Temminch, abondants au nord du neuvième parallèle, pourraient également jouer un rôle important.

\subsection{Genre Taenia Linné, 1758}

Cysticerques de Taenia regis (Baer, 1923)

Hôte: Phacochoerus aethiopicus (15 cas sur 70) (*).

Localisation: le plus souvent épiploon et mésentère; parenchyme hépatique; plus rarement muscles, cour et cerveau.

Répartition : 19.8.B.a. (un cas); 21.8.C.d. (un cas); 21.8.D.c. (4 cas); 21.8.D.d. (deux cas); 21.9.B.b. (trois cas); 22.9.A.c. (un cas) et C.a. (un cas); 25.5.D.d. (un cas).

Les lésions ont l'aspect de kystes de 9 à $20 \mathrm{~mm}$ de long sur 12 à $15 \mathrm{~mm}$ de large. Ils renferment un cysticerque en forme de cornue dont l'extrémité antérieure porte un scolex. Celui-ci est pourvu de quatre ventouses globuleuses, d'un rostellum non rétractile et d'une double couronne de crochets en forme de poignard, au nombre de 38 à 48 et placés en alternance. Les plus grands mesurent 240 à $280 \mu$ et les plus petits 150 à $173 \mu$.

Pendant longtemps, on a cru que le Cestode adulte correspondant était Taenia hydatigena (54). Actuellement, on pense plutôt qu'il s'agit de Taenia regis (Baer, 1923) (**) qui vit dans l'intestin du lion. Cette hypothèse qui a été émise dès 1967 par l'un d'entre nous (22) a été reprise récemment par plusieurs auteurs $(47,55)$.

Trois arguments viennent étayer cette hypothèse :

(*) Résultats définitifs.

(*) Taenia bubesei, Ortlepp, 1938, du lion africain a été mis en synonymie avec Taenia regis par Verster (55).
- d'ordre anatomique. Les dimensions du rostellum, le nombre total de crochets et la longueur des plus grands rapprochent étroitement Taenia regis des cysticerques de phacochères.

- d'ordre biologique: les essais d'infestation (55) du chat, du chien, du chacal et du lycaon à partir de cysticerques péritonéaux d'Artiodactyles sauvages sont tous demeurés négatifs.

- d'ordre géographique: en R.C.A., un lion tué sur la rivière Kapa (21.8.D.d.) hébergeait plusieurs Taenia regis. Or deux phacochères parasités ont été découverts au même endroit et cinq autres dans des zones proches (21.8.C.d. et 21.8.D.c.).

Dans ces conditions, il est tentant d'établir une relation formelle entre les Cestodes adultes du lion et les formes larvaires obtenues chez les phacochères. La preuve absolue fait, cependant, défaut, car seule l'infestation d'un lion avec des cysticerques de phacochères permettrait de lever définitivement le doute.

\section{TREMATODES $(* * *)$}

\section{ORDRE DES ECHINOSTOMIDA}

1. Famille des Gastrodiscidae Stiles et Goldberger, 1910

1.1. Genre Gastrodiscus Leuckart, 1877

Gastrodiscus aegyptiacus (Cobbold, 1876) Railliet, 1.893

Hôtes: Phacochoerus aethiopicus (24 cas sur 48); Hylochoerus meinertzageni (deux cas sur trois).

Localisation : gros intestin et caecum.

Répartition : toute la zone cynégétique.

Ce Trématode, commun aux Equidés, au rhinocéros et aux Suidés (11, 24), n'avait encore jamais été observé chez l'hylochère qui est donc un hôte nouveau.

Les infestations sont presque toujours importantes, même chez l'hylochère (l'un d'entre eux était porteur de 70 vers).

Rôle pathogène : on décrit classiquement des épisodes de coliques et de diarrhée chez les

(***;) D'après Baer (J.G.) et Joyeux (Ch.), in « Traité de zoologie » de Grassé, 1961, t. IV, fasc. I, 561-677. 
Equidés atteints de gastrodiscose. Dans le cas des Suidés sauvages, le parasite, jusqu'à plus ample informé, paraît bien supporté, malgré l'intensité des infestations.

1.2. Genre Choerocotyle Baer, 1959

Choerocotyle epuluensis Baer, 1959

Hôtes: Phacochoerus aethiopicus (un cas sur 48); Hylochoerus meinertzageni (deux cas sur trois).

Localisation : côlon.

Répartition : 25.5.D.d.

Ce parasite a été découvert pour la première fois par BAER (4) au Congo, chez un hylochère: c'est donc la seconde fois qu'il est rencontré en Afrique. On le trouve associé aux Gastrodiscus.

Chez l'unique phacochère parasité, il y avait environ 50 Gastrodiscus et, seulement, deux Choerocotyle.

Le phacochère est un hôte nouveau.

Rôle pathogène: inconnu, probablement identique à celui des Gastrodiscus.

2. Famille des Fasciolidae Railliet, 1895

Genre Fasciola Linné, 1758

Fasciola gigantica Cobbold, 1855

Hôte: Phacochoerus aethiopicus (un cas sur 48).

Localisation : canaux biliaires.
Répartition : 21.8.D.d.

Le matériel examiné comprend deux douves complètes et trois fragments.

Un grand nombre d'animaux de chasse, tels que girafes, buffles et diverses antilopes, sont susceptibles d'être parasités par Fasciola gigantica. Le phacochère est un hôte nouveau.

Dans la zone considérée, l'infestation des buffles est constante.

Rôle pathogène: Fasciola gigantica est un parasite adapté aux Bovidae. Lorsqu'il envahit le foie d'animaux appartenant à des familles différentes, ou bien il n'évolue pas, ou bien il provoque des troubles sérieux, même s'il n'est représenté que par un nombre restreint d'exemplaires. On peut donc admettre que, chez le phacochère, ce Trématode "accidentel " est pathogène.

\section{PENTASTOMIDA}

Ce parasitisme fera l'objet d'une note particulière englobant toutes les espèces animales infestées en République Centrafricaine.

Chez les Suidés sauvages, les larves enkystées se voient dans la cavité générale, plus rarement dans le foie ou la rate (en surface ou dans le parenchyme). Les kystes sont souvent calcifiés.

Le tableau suivant résume les observations faites :

\begin{tabular}{|c|c|c|c|}
\hline Espèce & Coord IBAH & Organe parasité & Nombre et état des kystes \\
\hline 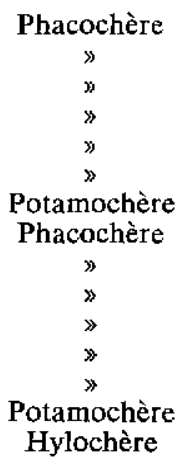 & $\begin{array}{c}\text { 20.7. C.b. } \\
» \\
» \\
» \\
» \\
\text { 20.7.D a. } \\
\text { 21.8.D.d. } \\
\text { 21.9.B.b. } \\
\text { 21.9.D.a. } \\
\text { 22.9.C.a. } \\
\text { 23.6.C.c. } \\
\text { 23.7.C.d. } \\
\text { 25.5.C.c. } \\
\text { 25.5 D.d. }\end{array}$ & $\begin{array}{l}\text { Surface diaphragne } \\
\text { Surface foie } \\
\text { Parenchyme hépatique } \\
\text { Rate. péritoine, coeur } \\
\text { Surface du foie } \\
\text { Parenchyme hépatique } \\
\text { Foie et mésentère } \\
\text { Fole et mésentère } \\
\text { Parench. hépat. et splénique } \\
\text { Foie, cavité abdominale } \\
\text { Surface du coeur et du foie } \\
\text { Parenchyme hépatique } \\
\text { Mésentère } \\
\text { Surface du foie } \\
\text { Surface du foie }\end{array}$ & $\begin{array}{l}\text { Un kyste } \\
\text { Deux kystes } \\
\text { Deux kystes calcifiés } \\
\text { Nombreux kystes } \\
\text { Deux kystes } \\
\text { Dix-sept kystes calcifiés ou non } \\
\text { Nombreux kystes } \\
\text { Nombreux kystes calcifiés ou non } \\
\text { Nombreux kystes } \\
\text { Nombreux kystes calcif. dans le foie } \\
\text { Nombreux kystes calcufiés ou non } \\
\text { Trois kystes calcifiés } \\
\text { Deux kystes calcifiés } \\
\text { Un kyste } \\
\text { Un kyste très petit et calcifié }\end{array}$ \\
\hline
\end{tabular}




\section{CONCLUSION}

Les 28 espèces d'helminthes $\left(^{*}\right)$ parasites des Suidés sauvages, rencontrées au cours de cette première enquête en République Centrafricaine, sont, pour les $4 / 5$, des parasites purement africains.

Les seules espèces cosmopolites sont: Stephanurus dentatus, Globocephalus urosubulatus, Physocephalus sexalatus, Gnathostoma hispidum et Setaria congolensis. Il s'agit là de parasites banaux des Suidés domestiques.

Stephanurus dentatus n'avait jamais été observé chez le phacochère, Globocephalus urosubulatus était à ce jour inconnu chez le potamochère et Setaria congolensis est une Filaire nouvelle pour l'hylochère.

Trois espèces sont communes à plusieurs familles d'hôtes. Ce sont :

- Deux Trématodes: d'une part, Gastrodiscus aegyptiacus qui parasite les Suidés et les Equidés, d'autre part, Fasciola gigantica que lon trouve chez les ruminants et, parfois, chez des hôtes très différents, tels que l'homme.

Le phacochère est un hôte nouveau pour Fasciola gigantica et, l'hylochère, pour Gastrodiscus aegyptiacus.

- Un Cestode, vraisemblablement Taenia regis, représenté sous sa forme larvaire (Cysticerque) qui existe en Afrique, non seulement chez les Suidés, mais encore chez de nombreux Artiodactyles de la famille des Bovidae.

Les autres helminthes (20 espèces) ne sont connus que chez les Suidés sauvages d'Afrique $(* *)$.

Quatre d'entre eux constituent des unités taxonomiques nouvelles et font l'objet d'études

(*) Sont exclus Echinococcus Sp. et les Pentastomida.

$(\because *)$ Nous sommes d'accord avec Le Roux (31) et Round (46) pour mettre en doute l'hôte, Hippotragus equinus, que Goodey (21) assignait, avec beaucoup de réserves, à Daubneya eurycephalum et Daubneya simpsoni. détaillées qui seront publiées ailleurs : ce sont quatre Nématodes appartenant aux genres Daubneya et Probstmayria. La grande variété des espèces d'Esophagostomes parasites des Suidés sauvages est, une fois de plus, confirmée. Désormais, l'hylochère doit être classé parmi les hôtes de Daubneya goodeyi, Daubneya yorkei et Daubneya mwanzae.

L'hylochère est également un hôte nouveau pour Murshidia (Chabaudia) pugnicaudata. Depuis les descriptions de Murshidia Chabaudia hamata et de Murshidia (Chabaudia) pugnicaudata, aucune autre espèce de ce genre, normalement parasite des grands herbivores, n'a été vue chez les Suidés. Il est bon de souligner aussi que ces vers n'ont jamais été recueillis chez le porc domestique.

Ascaris phacochoeri, Physaloptera joyeuxi et Moniezia mettami sont des helminthes classiques de la parasitofaune des phacochères; par contre, Choerocotyle epuluensis est nouveau, ainsi que Moniezia mettami pour l'hylochère.

Le hasard a voulu que les deux potamochères étudiés soient tous deux porteurs de parasites d'intérêt zoopathologique très grand (Globocephalus, Gnasthostoma, Echinococcus) et d'intérêt zoogéographique moindre, en ce qu'ils ne permettent pas une comparaison immédiate entre les faunes parasitaires des trois espèces de Suidés considérés.

Le présent travail rend surtout évidentes les relations qui existent entre le phacochère et l'hylochère. Celles-ci sont confirmées par la mammalogie : en effet, ces deux espèces sont les plus primitives de la famille des Suidae, tandis que le potamochère se rapproche davantage du sanglier (Sus scrofa).

Enfin, il est nécessaire d'insister sur le fait qu'aucun Suidé domestique n'est élevé dans la zone cynégétique, zone d'ailleurs extrêmement pauvre en installations humaines : on est donc en présence de l'une des rares régions du globe où le parasitisme des animaux domestiques n'interfère pas sur la faune helminthologique des animaux sauvages de genres voisins. 


\section{SUMMARY}

\section{Pathology of wild animals in Central Africa. Parasitism of wild Suidae. First report}

The authors record the parasitic species founded in the wild Suidae of the Central Africa. Four new species have been determined and described in an other paper.

The bushpig is a new host for one Nematoda species, the warthog for one Nematoda and two Trematoda species and the forest hog for five Nematoda, one Trematoda and one Cestoda species.

\section{RESUMEN}

\section{Encuesta sobre la patologia de la fauna salvaje en Africa Central. El parasitismo de los suidos salvajes. Primeros resultados de encuesta}

Los autores enumeran las especies parásitas de los suidos salvajes, recogidas en Africa Central, durante una encuesta sobre la patologia de la fauna cinegética.

Se determinaron y se describieron cuatro especies nuevas en una otra publicación.

El potamoquerio es un huesped nuevo para una especie de nemátodo, el facoquerio para una especie de nemátodo y dos especies de tremátodos, y el hiloquerio para cinco especies de nemátodos, una especie de tremátodo y una especie de céstodo.

\section{BIBLIOGRAPHIE}

1. ALESSANDRINI, Su di un raro parassita dell' intestino del maiale e sul genere Globocephalus Molin, Archs. Parasit., 1909, 13 : 458-76.

2. BAER (J.G.), Résultats zoologiques du voyage du Dr P. A. Chappius au Nil supérieur, Rev. suisse zool., 1923, 30: 337-52.

3. BAER (J. G.), FAIN (A.), Cestodes, Exploration du parc National de l'Upemba, Mission G.F. de Witte (1946-49), Brussels, 1955, fasc. 36, 38.

4. BAER (J. G.), Helminthes parasites. Exploration des Parcs Nationaux du Congo belge, Mission J. G. Baer - W. Gerber (1958), Brussels, 1959, fasc, $1,163$.

5. BAYLIS (H. A.), Notes on some parasitic Nematodes from East Africa, Ann. Mag. nat. Hist. $1920,9(6): 283-95$.

6. BAYLIS (H. A.), Report on a collection of parasitic Nematodes mainly from Egypt. Part. III : Camablidae ... with a note on Probstmavria and an appendix on Acanthocephala, Parasitology, 1923, 15: 24-38.

7. BAYLIS (H. A.), On two adult Cestodes from wild swine, Ann. Mag. nat. Histr. 1927, 19 : 417 25.

8. BAYLIS (H. A.), Notes on four Cestodes, $A n n$. Mag. nat. Hist., 1934, 14 : 587-94.

9. BAYLIS (H. A.), Nematodes from mammals West Africa, Ann. Mag. nat. Hist., 1936, 10 (7): 263-71, fig. 8-11.

10. BERGHE (L. VAN DEN), Enquêtes parasitologiques. II. Helminthes parasites, Exploration Parc national Albert, Mission Van den Berghe, Brussels, 1943, 2, 30.

11. BERNARDONI (J. L. C.), Contribution à l'étude des helminthes parasites du cheval au Niger. Thèse Méd. vét., Paris, 1969 , no 107, 71 p.

12. CHABAUD (A.), BAIN (O.) et HOUIN (R.), Nématodes de potamochères malgaches, Annis. Parasit. hum. comp., 1966, 41 (6), 599-606.
13. DAUBNEY (R.), A note on two species of the genus Murshidia (Nematoda strongyloidea) parasitic in the wart-hog, Ann. Mag. nat. Hist., 1923, 9 (11) : 256-63.

14. DAUBNEY (R.), Description of a new nematode Oesophagostomum mwanzee from the wart-hog, Ann. Mag. nat. Hist., 1924, 9 (13): 542-46.

15. DAUBNEY (R.), Esophagostomes from the wart-hog, Ann. Mag. nat. Hist., 1926, 9 (17) : 1117.

16. DESSET (M. C.), Contribution à la systématique des Filaires du genre Setaria; valeur des Dièrides, Mém. Mus. natn. Hist. nat., Paris, 1965, 39 (2): 257-87.

17. DIAOURE (A.), Strongylides parasites de mammifères du Congo-Brazzaville (Collect. R. Rousselot), Annls. Parasit. hum. comp., 1964, 39 (2) : 257-87.

18. DUTHY (B. L.), Three new Nematodes of the genus Oesophagostomum from the east african wart-hog, Phacochoerus aethiopicus, Ann. Mag. nat. Hist., 1947, 11 (14): 280-88.

19. FITZSIMMONS (W.M.), On Probstmayria reptiliae n. sp. from Homopus femoralis and some notes on the genus Probstmayria, J. Helminth., 1958,32 (4) : 211-18.

20. GEDOELST (L.), Notes sur la faune parasitaire du Congo belge, Rev, zool. af $r_{\text {. }}, 1916,5$ (1): 3.

21. GOODEY (T.), Some new members of the genus Oesophagostomum from the Roan antelope and the wart-hog, J. Helminth., 1924, 2 (3) : 135-48.

22. GRABER (M.), Rapp. a. 1966 Lab. Farcha, FortLamy, 1967, t. I, p. 242.

23. GRABER (M.), Pathologie de la faune sauvage du Tchad: premiers résultats d'enquêtes, Rapp. a. 1967 Lab. Farcha, Fort-Lamy, 1968, t. V, p. 95 .

24. GRABER (M.), Helminthes et helminthiases des équidés (ânes et chevaux) de la République du 
Tchad, Rev. Elev. Méd. vét. Pays trop., 1970, 23 (2) : 207-22

25. GRABER (M), EUZEBY (J.), et THAL (J.) Stephanurus dentatus Diesing, 1839 chez un phacochère. Phacochoerus aethiopicus, Pallas de l'est de la R.C.A., Bull. Soc. Path. exot., 1971. 64 (4) : 468-73.

26. GRETILLAT (S.), VASSLIADES (G.), Présence de Trichinella spiralis chez les carnivores et suidés sauvages de la région du fleuve Sénégal, $C . r$ hebd. Séanc. Acad. Sci. Paris, 1966, 264 (10): 1297-300.

27. JOYEUX (Ch.), GENDRE (E.), BAER (J. G.), Recherches sur les helminthes de l'A.O.F., Paris, Masson et Cie, 1928, 81 p. (Coll. Soc. Path. exot.).

28. KREIS (H. A.), Beiträge zur Kenntnis parasitischer Nematoden. XVIII. Das Genus Probstmayria Ransom, 1907, Schweizer Arch. Tierheilk. $1955,9(97): 422-33$.

29. KREIS (H. A.), Beiträge zur Kenntnis parasitischer Nematoden. XXVII. Wenig bekante parasitische Nematoden und zwei neue Filarien aus dem Warzenschwein - Phacochoerus aethiopicus, Schweizer Arch. Tierheilk., 1970, 7 (112), 324-36.

30. KOBULEJ (D. T.), Zur kenntnischer Anatomie und Systematik der Gattung Globocephalus Molin (Nematodes), Acta vet. hung., 1954, 4 (2/3) : 263. 73.

31. LE ROUX (P. L.), On the division of the genus Oesophagostomum Molin, 1861 into subgenera and the creation of a new genus for the Oesophagostomum of the wart-hog, J. helminth., 1940 , 18 (1) : $1-20$.

32. MAPLESTONE (P. A.), Nematodes parasites of pigs in Bengal, Rec. Indian Mus., 1930, 32 (2): 77-105.

33. MAPLESTONE (P.A.), Parasitic Nematodes obtained from animals dying in the Calcutta zoological gardens, Rec. Indian Mus., 1931, 33 (2) : 71-171.

34. MARLOW (B. J. G.), A commensal Nematode in the African Bush pig, J. Mammal., 1959, 36 (1): 144.

35. MOREL (P. C.), Les Helminthes des animaux domestiques de l'Afrique occidentale, Rev. Elev. Méd. vét. Pays trop., 1959, 12 (2) : 153-74.

36. NELSON (G.S.), RICKMAN (R.), PESTER (F. R.), Feral trichinosis in Africa, Trans. R. Soc. trop. Med. Hyg., 1961, 55 (6) : 514-17.

37. NEVEU-LEMAIRE (M.), Les CEsophagostomes des Phacochères, Annls. Parasit. hum. Comp., 1927, 5 (3) : 214-19.

38. ORTLEPP (R. J.). South African Helminths. Part. II. Some Taenias from large wild Carnivores, Onderstepoort J. vet. Res., 1938, 10 (2) 254-56.

39. ORTLEPP (R. J.), Observations on Helminths parasitic in wart-hogs and Bushpigs, Onderstepoort J. vet .Res., 1964, 31 (1): 11-38.

40. PETTER (A. J.), Equilibre des espèces dans les populations de Nématodes parasites du colon des tortues terrestres, Mém. Mus. natn. Hist. nat., Paris, 1966, 39 (1) : 1-252,

41. RAI (P.), AHLWALIA (S. S.), A note on Probstmayria vivipara (Probstmayr, 18-65) Ransom, 1907 , from the Indian pony, with a brief remarks on its systematic position, Proc. natn. Acad. Sci. India, 1958, 28 (6) : 395-98.
42. RAILLIET (A), HENRY (A), Sur une filaire péritonéale des porcins, Bull. Soc. Path. exot., 1911,4 (6) : 386-89.

43. RAILLIET (A.), HENRY (A.), Nematodes parasites du Congo Belge, Bull. Soc. Path, exot., 1918, 11 (2) : 82-98.

44. RAILLIET (A.), HENRY (A.), BAUCHE (J.), Un nouveau strongylidé du porc, Bull. Soc. Path. exot., 1919, 12 (6): 324-32.

45. RANSOM (B. H.), Probstmayria vivipara (Probstmayr, 1866) Ransom, 1907, a Nematode of horses heterotopore unreported from the United states. Trans. Am. microsc. Soc., 1907, $27: 33$.

46. ROUND (M. C.), Check list of the Helminth Parasites of African mammals, Commonwealth Bureau of Helminthology, St Albans, Technical Communication, 1968, no 38,252 p.

47. SACHS (R.), SACHS (C.), A survey of parasitic infestation of Wild herbivores in the Serengeti region in Northern Tanzania and the lake Rukwa in Southern Tanzania, Bull. epizoot. Dis. Afr., 1968, 16 (4) : 455-72.

48. SCHWARTZ (B.), Two new Nematodes of the family Strongylidae parasitic in the intestines of Mammals, Proc. U.S. natn. Mus., 1928, 73 (2): $1-5$.

49. SPASSKII (A. A.), Essentials of cestodology: Anoplocephalate Tapeworms of domestic and wild animals, Acad. Sci. U.R.S.S., Moscou, 1951, $730 \mathrm{p}$.

50. SPRENT (J. F.), Notes on Ascaris and Toxascaris, with a description of Baylisascaris gen. nov. Parasitology, 1968, 58 (1): 185-98.

51. THORNTON (H.), A review of the Oesophagostomes in the Collection of the Liverpool School of Tropical Medecine, Ann. trop. Med. Parasit., 1924, 18 (3) : 393-408.

52. TRONCY (P.M.), GRABER (M.), THAL (J.) Enquête sur la pathologie de la faune sauvage en République centrafricaine. Oesophagostominae des Suidés sauvages, Annls. Parasit. hum. comp. 1972 (à paraître)

53. TRONCY (P.M.), GRABER (M.), THAL (J.) Enquête sur la pathologie de la faune sauvage en République Centrafricaine, Probstmayria suis п. sp. Bull. Mus. natn. Hist. nat., Paris, 1972 (à paraître).

54. VAILLANT (L.), De la présence de Cysticercus tenuicollis chez le Phacochoerus africanus. $C . r$. Séanc. Soc. Biol., 1866, 17 (2): 91-93.

55. VERSTER (A.), A taxonomic revision of the genus Taenia Linnaeus 1758 , s. str. Onderstepoort J. vet. Res., 1969,36 (1): 3-58.

56. VUYLSTEKE (C.), Note sur quelques Nématodes parasites avec description de neuf espèces nouvelles, Rev. Zool. Bot. afr., 1956, 53 (3/4) : 44147.

57. WARREN (E. G.), Ascaris species in Wart-hogs, Annls. Parasit. hum. comp., 1970, 45 (2) : 251-52.

58. YAMAGUTI (S.), Systema Helminthum. The Nematodes of Vertebrates 1961, 3, Part. 2.

59. YEH (L. S.), A revision of the Nematode genus Setaria Viborg, 1795, its host-parasite relationship, speciation and evolution, J. Helminth., 1959, 33 (1) : 1-98.

60. YORKE (W.), MAPLESTONE (P.A.), The Nematodes parasites of Vertebrates, London, J. and A. Churchill, 1926, 536 p. 